\title{
Question Answering System of Management Philosophy based on Lecture Transcripts of Business Leaders
}

\author{
Hirotaka Mishina $^{1}$, Atsushi Aoyama ${ }^{2}$, Akira Maeda ${ }^{3}$ \\ ${ }^{1}$ Graduate School of Information Science and Engineering, Ritsumeikan University, Japan \\ ${ }^{2}$ Graduate School of Technology Management, Ritsumeikan University, Japan \\ ${ }^{3}$ College of Information Science and Engineering, Ritsumeikan University, Japan
}

\begin{abstract}
In recent years, a growing number of companies have been suffering damages due to the occurrence of frauds or illegal activities by their own employees and losing their trust in various companies including some large companies. These things are thought to happen due to the lack of corporate rules and philosophy concerning management. Based on these circumstances, in order to answer the questions on how to manage companies, this paper proposes a question answering system for company managers using lecture transcripts of Dr. Kazuo Inamori, who is one of the most respected business leaders in the world. In the proposed system, we analyze the managers' questions, extract answers to these questions from the lecture transcripts, and return it to the users.
\end{abstract}

Keywords: Kazuo Inamori, non-factoid question answering, management philosophy

\section{Introduction}

Digital archiving of respected business leader's management philosophy could be a useful learning tool for people in the position of management not only in companies but also in other organizations such as educational institutions and governments. In this paper, we propose a non-factoid question answering system focusing on management philosophy using the digital archive of lecture transcripts of one of the most respected business leaders in Japan, Kazuo Inamori.

\section{The Proposed Method}

In question answering systems, we first have to identify the type of the user's question. We set three question types, i.e., "Why", "How", and "What". We use Support Vector Machine (SVM) as the method for identifying the question type. The identification is made by multiclass classification using one-versus-rest method. The next step is to retrieve the documents which contain the answer to the question. Query expansion using word2vec[1][2] is performed on nouns extracted from the user's question sentences. The TF-IDF values of the query including the terms expanded by word $2 \mathrm{vec}$ are calculated, and the sum of the TF-IDF values for a document is taken as the score, and the 
top 20 documents are taken as the answer candidate documents. From these documents, sentences which can be considered as answers to the question are extracted from the sentences of each candidate document. Finally, the cosine similarities between vectors of nouns in the question sentences and nouns in the answer candidate sentences are calculated, and the top-ranked sentences are returned to the user.

\section{Experimental Results}

We conducted an experiment to validate the effectiveness of the proposed question answering system. 10 questions were used in the experiment. The results of one of these questions are listed in Table 1. In this experiment, we output 20 sentences. Whether the answer was appropriate for the question was judged by the first author. In this case, the answers shown in Table 1 were regarded as appropriate for the question.

Table 1. An example of a question and extracted answers (excerpt) in the experiment

\begin{tabular}{|l|l|}
\hline Question & Why do we need altruism in management? \\
\hline \hline $\begin{array}{l}1 \text { st } \\
\text { answer }\end{array}$ & $\begin{array}{l}\text { If you expect good results, it is essential that you do business with the } \\
\text { heart of mind and caring heart. }\end{array}$ \\
\hline $\begin{array}{l}\text { th } \\
\text { answer }\end{array}$ & $\begin{array}{l}\text { If I decide things only with your mind's thought, your will harm the } \\
\text { society, and will cause serious inconvenience to working employees. }\end{array}$ \\
\hline $\begin{array}{l}11 \text { th } \\
\text { answer }\end{array}$ & $\begin{array}{l}\text { Strong self-interest is indispensable for doing business. We have to } \\
\text { make sure to give altruism. }\end{array}$ \\
\hline
\end{tabular}

\section{Conclusion and Future Work}

In this paper, we proposed a question answering system of management philosophy using the business leader's lecture transcripts. In future work, we are planning to incorporate deep learning methods in ranking responses, searching documents, and extracting answer candidates. Besides, in the current document retrieval phase, only the words in question sentences are used. In the future, we will pay attention not only to words but also to syntax, and the characteristic words of Inamori could be considered for special processing such as weighting. Regarding the answers, the user intends to select the most helpful answer from the ranked results, therefore incorporating a mechanism for the system to learn the answers chosen by the users could improve the effectiveness of the answer selection process. In the current experiment, we made judgments whether the answers were appropriate for the question by the first author, but we plan to make judgments by consulting Kazuo Inamori's books that may contain the correct answers.

\section{References}

1. Tomas Mikolov, Ilya Sutskever, Kai Chen, Greg Corrado, Jeff Dean: Distributed representations of words and phrases and their compositionality, Proc. NIPS2013 (2013)

2. Word2vec, https://code.google.com/p/word2vec/ 